\title{
Use of phasor measurements for modeling FACTS at the electric power system state estimation
}

\author{
Irina Kolosok ${ }^{1}$, Elena Korkina, ${ }^{1, *}$, and Aleksandr Tikhonov ${ }^{1}$ \\ ${ }^{1}$ Energy Systems Institute by L.A.Melentiev, Siberian Branch of the Russian Academy of Sciences, Irkutsk, Russia
}

\begin{abstract}
As the Smart Grid is being developed in the Russian Federation, a need in FACTS arises at resolving the issues of increasing the transmission capacity of electric power lines, voltage control, etc. The paper considers the issues of installing phasor measurement units (PMU) at the FACTS for the electric power system state estimation of (EPS SE). The relevance of the paper is explained by the mandatory introduction of PMUs at newly commissioned power engineering facilities (PEF) scheduled for 2020 and farther. PMUs registering voltage and current phasors at FACTS allow increasing the precision of results of the EPS SE problem and improving the electric modes at the considered PEF.
\end{abstract}

\section{Introduction}

Smart Grid making stipulates for the development of organization and structural solutions in mode management as well as the upgrade of available grids for voltage regulation, increase the transmission capacity of electric power lines, etc. The actions on EPS upgrade are conducted, in particular, through the installation of controllable (flexible) systems of AC transmission FACTS. FACTS allows managing not only certain parameters of electrotechnical equipment but a group of parameters in dynamics $(U, \delta, P$ and $Q$ ).

Transition to Smart Grid is not possible without introducing the wide area monitoring system (WAMS) [1]. PMUs with high precision register the measurements of state parameters. WAMS development in Russia gains its pace: in 2005 only two foreign PMUs were installed while in 2018 at the EP facilities 715 PMU were installed (mostly produced in Russia) [2]. A system operator of the Unified Power System plans the PMU mandatory installation at newly commissioned EP facilities starting from 2020. As it is stated in [3], PMU is the only today's technology allowing for the monitoring of dynamic processes in real time.

Dispatching control practice most frequently solves two problems to form a model of the current state: load flow calculation (LFC) and the EPS SE based on the measurements of SCADA and WAMS [4]. As of today SCADA is a modern software complex collecting and transferring data from the facilities to a central two-way station (CTWS); for remote control of electrotechnical equipment at the facility; for control algorithm implementation; for identifying the emergency state and informing the dispatching staff at the electric power company. The measurement system SCADA, applied today, cannot provide sufficient basis for making a comprehensive Smart Grid that would correspond to all the requirements set to "smart grids", including those relating to FACTS (being its main component) control. It is explained by insufficiently high discretization of transmitted signals as well as their reliability as there are limitations existing for signal transmission from the sensors to CTWS because of the system architecture and algorithms. In addition, one SCADA measuring device registers only a small number of parameters - it is not suitable in case of FACTS.

One of the possible solutions of the problem is applying PMU allowing for registering virtually all FACTS parameters with a high discretization level at continuous data reading. This allows implementing the algorithms of FACTS control at the facilities and transmit the information for forming control actions to the upper level (the dispatching center) taking into account the mode and circuit state of EPS. The tandem use of FACTS and PMU allows preventing, controlling and localize system incidents with minimum aftermath for EPS operation.

Both abroad and in Russia primarily the research were conducted on inclusion the FACTS models in the LFC problem [5-9], and later - in the the EPS SE problem [10-15]. [12] discusses a limited transmission capacity of electric power lines (EPL) and involving FACTS into the SE problem. [13] suggests a state estimation algorithm in power systems where the FACTS and PMUs are located. Russian papers [14, 15] of 2012 suggest a two-stage procedure of local SE for EPL with the account of FACTS the results of which confirm the efficiency of the PMU application at the validation of grid and FACTS parameters. [15] suggests local SE on the basis of STATCOM. The measurements at the EPL ends including a synchronous static compensating device (STATCOM) allow estimating not only the electric state parameters but also the parameters of transmission equivalent circuits that significantly

\footnotetext{
Corresponding author: korkina@isem.irk.ru
} 
reduces the EPS SE resulting error. Unfortunately, in those years, the number of PMUs was insufficient and the problem was not resolved. Now several FACTS are installed in Russia. Not all of them are equipped with PMU.

In 2016 Melentiev ESI SB of RAS conducted a largescale work on FACTS modeling in the EPS SE algorithms [16-21]. In this paper the authors present the models of controlled shunt reactors (CSR), phaseshifting transformer (PST) and HVDC included in the SE problem. To increase the grid observability, equipping these models with PMUs is suggested. It is shown that the solution of the SE problem with the inclusion of FACTS equipped with PMUs provides a significant improvement of quality of measurement estimates.

\section{Modeling FACTS at solving the problem of EPS state estimation}

\subsection{The EPS state estimation problem}

The EPS SE problem consists in calculating the EPS steady-state conditions based on measurements $\bar{y}$ [2]. State estimation algorithms traditionally deals with SCADA: voltage magnitudes $U_{i}$, nodal power injections $P_{i}, Q_{i}$, power flows in lines $P_{i j}, Q_{i j}$, more rare - nodal and line currents $I_{i}, I_{i j}$. So, measurement vector looks like:

$$
\bar{y}=\left(P_{i}, Q_{i}, P_{i j}, Q_{i j}, U_{i}, I_{i}, I_{i j}\right)
$$

Since WAMS has been created a new generation of measurements started

$$
\bar{y}=\left(\dot{U}_{i}, \dot{I}_{i}, \dot{I}_{i j}\right)
$$

with synchronized phasor measurements of nodal voltage $\dot{U}_{i}=U_{a i}+j U_{r i} ;$ nodal $\dot{I}_{i}=I_{a i}+j I_{r i}$ and line currents $\dot{I}_{i j}=I_{a i j}+j I_{r i j}$.

For solving the EPS SE problem the Test Equation method was developed in MESI SB RAS [4]. Test Equations (TE) are the steady state equations that consist of only measurements $\bar{y}$ :

$$
w_{k}(y)=0
$$

When using the TE method the EPS SE problem concludes in minimization of a function value:

$$
J(y)=(\bar{y}-\hat{y})^{T} R_{y}^{-1}(\bar{y}-\hat{y})
$$

subject to constraints in the form of steady state equations (3). In (4), $R_{y}$ - the covariance matrix of measurement errors. At the second stage, based on estimates of basic measurements, estimates of the state vector (complexes of nodal voltages) are calculated then line power flows and nodal power injections are calculated.

\subsection{PMU arrangement at EPS facilities}

The problem of PMU location is well-known to the researchers and designers occupied with the issues of PMU application in power engineering. To provide for correct operation, all PMU are time-synchronized by means of signal receivers of global positioning systems (GPS, GLONASS). Each variant of PMU installation aimed at some specific purposes:

- EPS facilities of special significance [2],

- the boundary nodes of adjacent EPS at the decomposition of the SE problem [22],

- the nodes improving the properties of the EPS SE problem (criteria redundancy increase; eliminating critical measurements and groups) [23],

- at calculating the linear SE in the areas which can be fully observed by PMUs $[1,24]$,

- the substations with FACTS [25-29, etc.]

Semiconducting technology development in 90 -s of the XXth century allowed making turn-off thyristors (GTO and GCT), fast diodes and insulated-gate bipolar transistor (IGBT) operating in the voltage range of 2.5$6 \mathrm{kV}$, with cutoff currents 1,500-4,000A. This breakthrough facilitated the invention of a new type of converters - voltage converters (VC) - due them modern FACTS of the second generation were made (for example, PST and HVDC on the voltage controller basis).

\subsection{PMU arrangement on FACTS models at the EPS state estimation}

To demonstrate PMU arrangement at the approbation of the PST, CSR, HVDC, the authors use the $500 \mathrm{kV}$ grid of the real power system (19 nodes, 28 lines) (Fig.1) [21]. The Northern region of this system locating the largest electric power sources is redundant while the Southern is deficient. The lines 7-8, 8-9 are loaded for a nominal power that leads to significant losses of electric power in them (approximately $50 \%$ of all power losses of that power system).

\subsubsection{Modeling a phase-shifting transformer}

PST is used to control power flows by changing voltage angles at the line ends (Table 1, Fig. 2,a, line 3-1). The traditional PST structure uses a cascade of two transformers - an regulated transformer $\mathrm{T} 1$ connected directly to a bus supplying the line on the part of the power source and a series transformer T2 (Table 1, Fig. $2, b)$ which is connected in series to overhead line by means of a secondary winding [21]. By means of regulating voltage at $\mathrm{T} 1$ the authors change the voltage magnitude and angle at $\mathrm{T} 2$ thus changing the power flows transferred in the line.

To model a booster transformer $\mathrm{T} 2$ with the capacity of $630 \mathrm{MVA}$, the authors include the node 20 and line 320 in the network while to model a reducing transformer T1 and PN2 - the node 21 and the line 3-21. To initially set PST and further control its operation as well as to increase its observability, it is suggested installing PMU in the node to which a series transformer is connected, i.e., in the node 3 . 


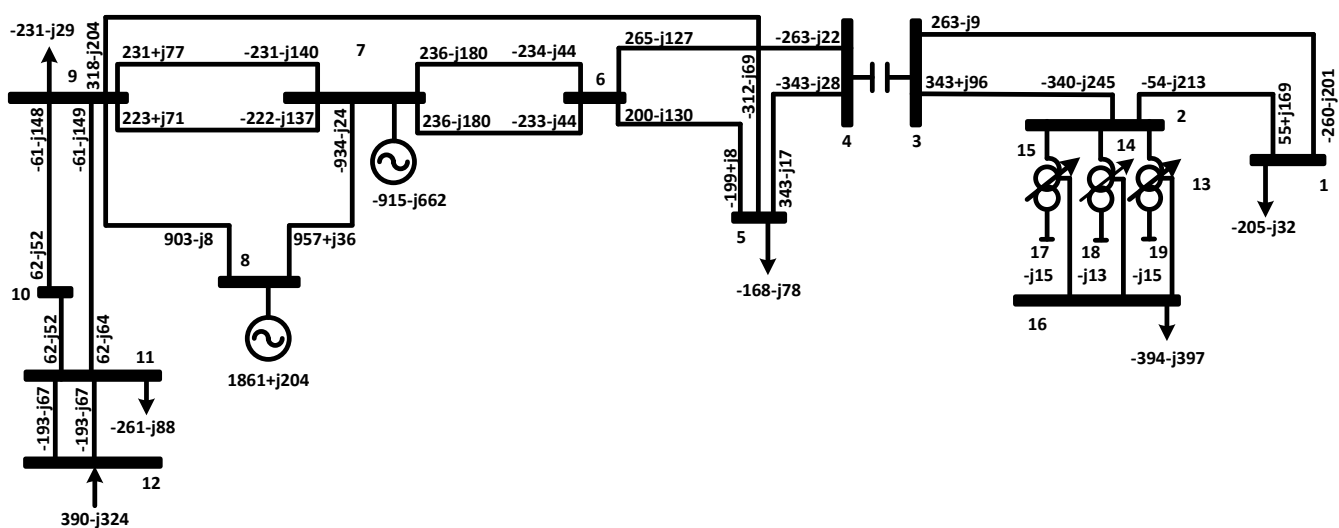

Fig. 1. 19-nodal circuit of power system

As it is clear from Table 1, PMU installation increase the observability of the network while the measurement estimates in the node 3 and incident lines become more precise. In the basic mode along the line 3-1 an "excess" active power is transmitted. It exceeds the load required in the node 1 , while along the line 3-2 the power insufficient for load supply in the node 2 . Along the line 1-2 an additional (reversive) power flows from the node
1 to the node 2. When inclusing Phase-Shifting Transformer the active power flow in the line 3-2 increased from $343 \mathrm{MW}$ to $397 \mathrm{MW}$ while in the line 31 it decreased from $262 \mathrm{MW}$ to $208 \mathrm{MW}$. A total power in the node 3 virtually did not change $(-606 \mathrm{MW})$ while the power flow towards the node 1 is virtually equal to zero.

Table 1. Phase-shifting transformer modeling

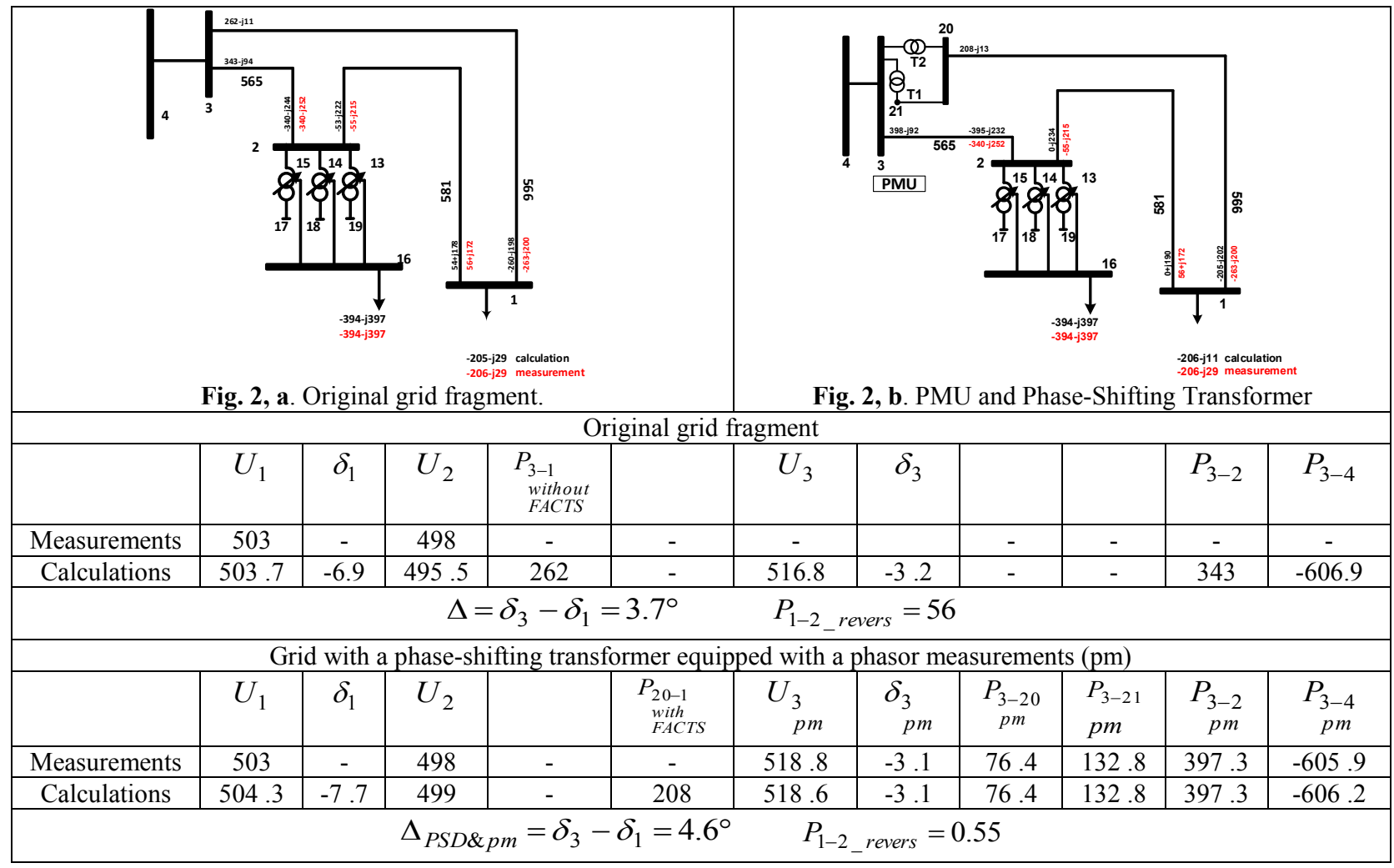

\subsubsection{HVDC modeling in the EPS SE problem}

HVDC are applied for a coordinated operation of AC and DC power electric grids; for the grids with various electric current frequency; for improving the transmission capacity of grid components containing "weak" links [31]. HVDC can be presented by two voltage sources [8]. Each voltage source is connected to the AC system via a transformer. HVDC is modeled at the substation in the node 12 (Table 2, Fig.3,a) to which one connects two branches modeling the transformer 12112 and the rectifier 112-1112. To connect an inverter to the circuit, the authors add the node 122 and two lines modeling the transformer 22-122 and the inverter 1221122. For HVDC the authors introduce an additional equation describing the process of active power transfer via $\mathrm{HVDC}$, i.e. the active power equality $P_{\text {rectifier }}$, consumed by the rectifier and $P_{\text {inverter }}$ provided by the inverter: 


$$
P_{\text {rectifier }}+P_{\text {inverter }}=0
$$

The equation (5) is considered as a test equation at

Table 2. HVDC modeling

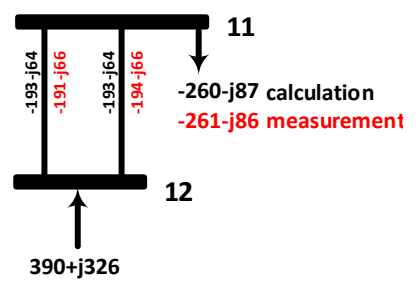

Fig.3, a. Original network fragment

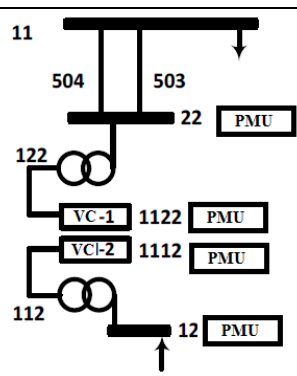

Fig.3, b. PMU and HVDC on the basis of voltage converter

\begin{tabular}{|l|l|l|l|l|l|l|l|l|l|l|l|l|l|}
\hline & $U_{12}$ & $\delta_{12}$ & $P_{1112}$ & $Q_{1112} P_{1122}$ & $Q_{1122}$ & $U_{1112} \delta_{1112}$ & $U_{1122}$ & $\delta_{1122} U_{22}$ & $\delta_{22}$ \\
\hline Meas. & 512 & - & -391 & 85 & - & - & - & - & - & - & 512 & - \\
\hline Calc. & 512 & 0 & -391 & 85 & 392.8 & -187.6 & 11 & -10.8 & 9.3 & 25 & 511.8 & 11.8 \\
\hline \multicolumn{8}{|c|}{ Network with a HVDC on the basis of voltage converter equipped with PMU } \\
\hline \\
\hline
\end{tabular}

At making the model of HVDC on the voltage converter basis undesirable problems of measurement lack occur. In practice PMUs are installed at voltage converters that is why the nodes 1112 and 1122 were selected for this purpose. In addition, two PMU are installed in the nodes 12 and 22 (Table 2, Fig.3,b). As it is clear from Table 2, the obtained estimates of voltages are equal to the measurement of voltages in the nodes where PMUs are installed and, due to more precise voltage, the authors specified the reactive power estimate.

Table 3 shows that the condition (5) at the transfer of active power on HVDC is satisfied $\left(P_{1112}+P_{1122}=0\right)$.

Table 3. HVDC "cut-in" conditions

Rectifier PN2 emits active power in the grid and gets reactive power

\begin{tabular}{|c|}
\hline$U_{1112}>U_{112}(11.4 \mathrm{kV}>11.0 \mathrm{kV})$ \\
\hline$P_{1112}=-391 \mathrm{~kW} ; \quad Q_{1112}=166$ MVar \\
\hline $\begin{array}{l}\text { Inverter PN1 receives active power from the grid and } \\
\text { puts out reactive power } \\
\qquad U_{1122}<U_{122}(9.3 \mathrm{kV}<10.0 \mathrm{kV}) \text {. }\end{array}$ \\
\hline$Q_{1122}=-190 M$ Var. \\
\hline
\end{tabular}

\subsubsection{Modeling a controlled shunt reactor (CSR).}

Some time ago two uncontrolled shunt reactors (180MVar each) (Table 4, Fig.4,a) were installed at the $500 \mathrm{kV}$ substation (node11). They did not allow smooth compensation of excess reactive power occurring at the substation due to traction load at the side of $110 \mathrm{kV}$ of that substation and the availability of system connecting overhead lines of $500 \mathrm{kV}$ with the power system of neighbour area. To provide smooth load control, in 2012 one of SR was replaced by a controlled shunt reactor (CSR). The CSR, providing a smooth control of inductance, allows maintaining a required voltage level in the overhead line node connection with a large charge capacity. At the use in the EPS SE problem of the CSR model with the control angle the CSR is modeled by a variable conductivity in the installation node the value of which is defined during calculation. The node voltage is registered. Depending on the obtained conductivity value, one can define the thyristor ignition angle. To improve the CSR observability, one should install PMU at the bus where CSR is installed (node 111) (Table 4, Fig.4,b).

\section{Conclusion}

Modern means for measuring the parameters of electrical power system modes (PMU, digital measurement devices) and electrical power management units (FACTS, energy accumulators, etc.) significantly increase the observability and controlability of electrical power systems. The solution of the EPS SE can be more precise and efficient due to the application of the precise measurements of WAMS. 
Table 4. Modeling a controlled shunt reactor (CSR).

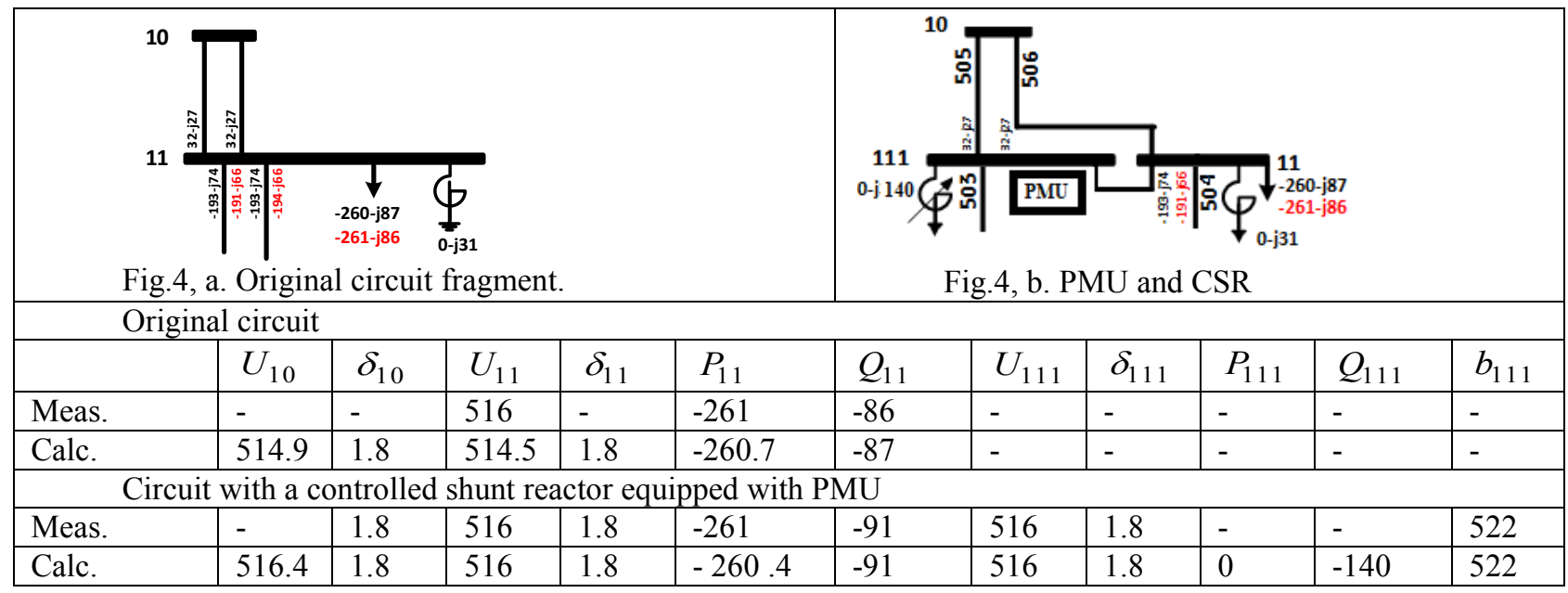

The PMU provides for the precise setting of FACTS functioning and subsequent monitoring. The calculations conducted for the section of the $500 \mathrm{kV}$ grid of the Irkutsk energy system proved the correctness of the results obtained. This makes it possible to adopt the data for the electric power system state estimation in real life.

The research was carried out under State Assignment, Project III.17.4.2 (reg. № AAAA-A17-117030310438-1) of the Fundamental Research of Siberian Branch of the Russian Academy of Sciences.

\section{References}

1. A. Phadke, J. Thorp. Synchronized phasor measurements and their applications. (Springer, NewYork, 2008)

2. A.V. Zhukov Experience of applying WAMS technology for problems of monitoring and control in power engineering. Report at the meeting of Noncommercial Partnership Science\&Technical Council of Uniform Power Grid. 10.10.2018

3. Prospects of phasor measurement application// http://digitalsubstation.com/blog/2018/05/07/perspe ktivy-primeneniya-sinhronizirovannyh-vektornyhizmerenij/

4. A. Gamm, A. Glazunova, Yu. Grishin, I. Kolosok, E. Korkina. Electricity, 6 (2009).

5. N.Hingorani, L.Gyugyi. Understanding FACTS concepts and technology of flexible AC transmission systems (IEEE Press, 2000)

6. E. Acha, C.R. Fuerte-Esquivel, H. Ambriz-Perez, C. Angeles-Camacho. FACTS. Modelling and Simulation in Power Networks. (John Willey\&Sons, England, 2004)

7. V.Kochkin. News of Electrotechnics, 4(46), (2007)

8. N.Voropai, A.Osak. Development of Equipment and Control Systems for Large Power Engineering Systems. Science\&Technology Report, 2008-0-2.731-01-007. Irkutsk, 2009

9. P.Yerokhin, V.Neuimin et al. News of Science\&Technical Center of Uniform Power Grid, 66(1), 2012

10. T. Okon, K. Wilkosz. PowerTech'2011, Norway.

11. E. Zamora-Cárdenas, B.Alcaide-Moreno, C.FuerteEsquivel. EPSR, 81 (2011)
12. J. Kumar, N. Kumar. Intern. Conf. on Recent Advances in Management, Science, Technology, Education and Legal Issues, 2019.

13. V. Presada, C. Cristea, M. Eremia, L. Toma. UPEC'2014. doi: 10.1109/UPEC.2014.6934712.

14. P.Bartolomey, S.Eroshenko, E.Lebedev, A.Suvorov. The 3d Intern. Science\&Technology Conf., 2 (2012)

15. P.Bartolomey, S.Eroshenko, E.Lebedev, A.Suvorov. IEEE PES ISGT, Germany, 2012.

16. I.Kolosok, A.Tikhonov. Methodological problems in reliability study of large energy system. Minsk, 66 (2015)

17. I.Kolosok, A.Tikhonov. Industrial Power Engineering, 10 (2015)

18. I.Kolosok, A.Tikhonov, E.Korkina. Methodological problems in reliability study of large energy system. Syktyvkar, 67 (2016)

19. A.Tikhonov. Thesis (Melentiev ESI SB RAS, Irkutsk, 2017)

20. I.Kolosok, A.Tikhonov, E.Korkina, A.Mahnitko, $\begin{array}{ll}\text { A.Gavrilovs. } & \text { doi: }\end{array}$ /RTUCON.2018.8659809.

21. I.Kolosok, A.Tikhonov. Methodological problems in reliability study of large energy system. Irkutsk, 69 (2018)

22. I.Kolosok, E.Korkina, A.Paltsev. PowerTech'2011, Norway.

23. A. Gamm, A. Glazunova, I. Kolosok, E. Korkina. DRPT'08, China.

24. I.Kolosok, E.Korkina, E.Buchinsky. PSCC-2014, Poland.

25. Yang Guangya, M.Gordon, A.Nielsen, J. Østergaard. Future Energy Systems Workshop, 2009.

26. B.Singh, N.Sharma, A.Tiwari, K.Verma, S.Singh. MultiCraft Intern. Journ. of Engineering, Science and Technology, 3(3) 2011

27. M.Dalali, H.Kargar. PSPC2015, Tehran.

28. M. Khan and A.Sh. Siddiqui Perspectives in Science, 8 (2016)

29. E.Zamora-Cárdenas,C.Fuerte-Esquivel, A.PizanoMartínez, H.Estrada-García, EPSR, 133 (2016).

30. Edris R. A., Bake M. H. et al. IEEE Trans. on Power Delivery, 12(4) (1997)

31. HVDC. http://www.ntcpower.ru/innovative_projects/insert_dc/ (access date: 27.02 .2018 ). 